\title{
In Situ Transmission Electron Microscopy Study on the Reaction Kinetics of the Ni/Zr-interlayer/Ge System
}

\author{
Jae-Wook Lee, Jee-Hwan Bae, Tae-Hoon Kim, Keesam Shin ${ }^{1}$, Je-Hyun Lee ${ }^{1}$, \\ Jung-il Song ${ }^{2}$, Cheol-Woong Yang* \\ School of Advanced Materials Science \& Engineering, Sungkyunkwan University, Suwon 440-746, Korea \\ ${ }^{1}$ School of Nano and Advanced Materials Engineering, Changwon National University, Changwon 641-773, Korea \\ ${ }^{2}$ Department of Mechanical Engineering, Changwon National University, Changwon 641-773, Korea
}

*Correspondence to: Yang $\mathrm{CW}$,

Tel: +82-31-290-7362

Fax: +82-31-290-7371

E-mail: cwyang@skku.edu

Received March 16, 2015

Revised March 23, 2015

Accepted March 23, 2015

\begin{abstract}
The reaction kinetics of the growth of $\mathrm{Ni}$ germanide in the $\mathrm{Ni} / \mathrm{Zr}$-interlayer/Ge system was investigated using isothermal in situ annealing at three different temperatures in a transmission electron microscope. The growth rate of $\mathrm{Ni}$ germanide in the $\mathrm{Ni} / \mathrm{Zr}$ interlayer/Ge system was determined to be diffusion controlled and depended on the square root of the time, with the activation energy of $1.04 \pm 0.04 \mathrm{eV}$. For the Ni/Zrinterlayer/Ge system, no intermediate or intermixing layer between the $\mathrm{Zr}$-interlayer and Ge substrate was formed, and thus the Ni germanide was formed and grew uniformly due to Ni diffusion through the diffusion path created in the amorphous Zr-interlayer during the annealing process in the absence of any intermetallic compounds. The reaction kinetics in the $\mathrm{Ni} / \mathrm{Zr}$-interlayer/Ge system was affected only by the $\mathrm{Zr}$-interlayer.
\end{abstract}

Key Words: Kinetics, Ni germanide, Zr-interlayer, Isothermal annealing, In situ transmission electron microscopy

\section{INTRODUCTION}

Germanium $(\mathrm{Ge})$ is one of the first materials to receive wide attention for use in semiconductor device fabrication, and $\mathrm{Ge}$ metal oxide semiconductor field effect transistors (MOSFETs) are considered to be promising devices for future high-speed complementary metal-oxide-semiconductor technology due to their high carrier mobility. It is well known that self-aligned germanide (salmanide), like self-aligned silicide (salicide), is effective in reducing the parasitic source/drain resistance. Germanide can be formed by a solid-state reaction between a metal and Ge. Among the various metal germanides, $\mathrm{Ni}$ germanide is being considered as the most promising salmanide material for Ge MOSFET technology due to its low formation temperature, only a nickel mono-germanide phase and low sheet resistivity (Balakrisnan et al., 2005; Hsu et al.,
2005; Zhang et al., 2005; Zhu \& Nakajima, 2005). However, one challenge of $\mathrm{Ni}$ germanide is its low thermal stability due to agglomeration and/or penetration of Ni germanide itself (Zhang et al., 2008). There have been many attempts to improve the thermal stability of Ni germanide by addition of alloying element or interlayer. In our previous studies (Lee et al., 2007, 2008, 2013), the formation and microstructural evolution of the $\mathrm{Ni}$ germanides formed in $\mathrm{Ni} /$ metal-interlayer/ Ge systems, i.e., Ni/Ta-interlayer/Ge and Ni/Zr-interlayer/ Ge systems, were examined as a function of annealing temperature in ex situ and in situ annealing experiments. It was found that the sheet resistance values of the germanides formed in the $\mathrm{Ni} /$ metal-interlayer/Ge systems remained stable at lower values than those in the $\mathrm{Ni} / \mathrm{Ge}$ system at high temperatures. An improvement in the thermal stability of the Ni germanide was caused by a reduction in the surface

This work was supported in part by the NRF grants funded by the Korea government (MSIP) (No. 2011-0019984 and No. 2011-0030058) and in part by grants (No. 10041134 and No. 10039200) funded by the Ministry of Trade, Industry \& Energy of the Republic of Korea.

@ This is an open-access article distributed under the terms of the Creative Commons Attribution Non-Commercial License (http://creativecommons.org/licenses/by-nc/3.0) which permits unrestricted noncommercial use, distribution, and reproduction in any medium, provided the original work is properly cited.

Copyrights $\odot 2015$ by Korean Society of Microscopy 
free energy due to the metal-rich layer formed on top of the newly formed Ni germanide film. However, in order to fully understand the effects of interfacial phenomena on the thin film reaction, it is important to find the kinetic parameters for the growth of Ni germanide and the thermodynamic information about these systems. One of the many advantages of in situ transmission electron microscopy (TEM) is that the kinetic parameters can be obtained simultaneously with a direct observation of the reactions.

In this study, the reaction kinetics of the growth of $\mathrm{Ni}$ germanide in the Ni/Zr-interlayer/Ge system was examined. In order to obtain the kinetic parameters of the systems, isothermal in situ annealing experiments were carried out in a TEM equipped with a specimen-heating holder. The variation in the thickness of the Ni germanide layer during annealing at different temperatures as a function of annealing time was measured using the TEM micrographs.

\section{MATERIALS AND METHODS}

A p-type $\left(\begin{array}{lll}1 & 0 & 0\end{array}\right)$ Ge wafer was cleaned sequentially with trichloroethylene, acetone, methanol, deionized water, and a dilute hydrofluoric acid (HF) solution ( $\left.\mathrm{HF}: \mathrm{H}_{2} \mathrm{O}=1: 100\right)$, and was immediately loaded into a DC magnetron sputtering system. Then approximately $1 \mathrm{~nm}$-thick $\mathrm{Zr}$ and $15 \mathrm{~nm}$-thick Ni films were deposited sequentially at room temperature under Ar gas pressure of $0.5 \mathrm{~Pa}$ after reaching a base pressure below $2.0 \times 10^{-5} \mathrm{~Pa}$. Cross-sectional high resolution (HR)TEM micrograph of the as-deposited specimen of the $\mathrm{Ni} / \mathrm{Zr}$ interlayer/Ge system formed by this way is shown in Fig. 1 . For TEM analysis, conventional ion-milled specimens were

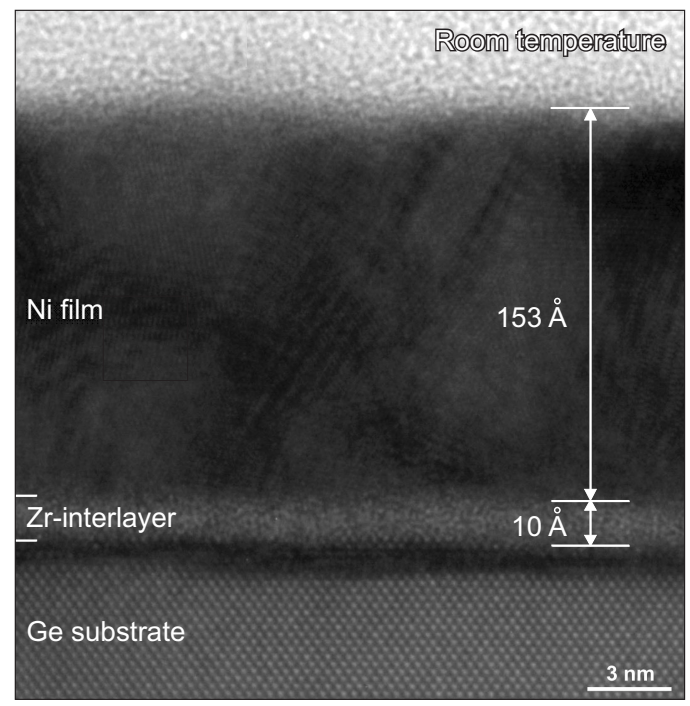

Fig. 1. Cross-sectional high resolution transmission electron microscopy micrograph of Ni film and $\mathrm{Zr}$-interlayer deposited on Ge substrate observed at room temperature. prepared by mechanical grinding using a tripod polisher (model 590; South Bay Technology Inc., USA) followed by Arion milling in a precision ion polishing system (PIPS; Gatan Inc., USA). In particular, the TEM specimens were fixed on Mo oval grids that can provide the high thermal stability required for the in situ annealing experiments.

One of the important experimental parameters in the in situ annealing experiment in a TEM is the temperature of a specimen which is conventionally measured by thermocouple. As the thermocouple of the specimen-heating holder is not in direct contact with the specimen, the measured temperature may be different from the temperature of the area of interest in the TEM specimen. Therefore, in order to observe the reaction of the specimen according to temperature, it is important to measure the actual temperature of the specimen during an in situ heating experiment. This can be achieved by the calibration experiment with pure element specimens. In this study, the calibration curves were obtained using pure $\mathrm{Sn}$ and pure Al. The detailed procedure of the calibration experiment is described in elsewhere (Lee et al., 2015).

The other important experimental parameter is a dimension such as thickness of the thin film layers, length and width of nanostructures, etc. It can be determined easily and accurately using digital TEM images. In order to measure the thickness of a thin film, it is essential to calibrate the size of the digital image at each magnification. Therefore, we perform this calibration at each magnification for the UltraScan CCD camera (USC 1000; Gatan Inc.) attached to the TEM (JEM3011; JEOL Ltd., Japan) using the $\mathrm{MAG}^{\star} \mathrm{I}^{\star} \mathrm{CAL}^{\circledR}$ calibration reference standard (SPI Supplies, USA).

\section{RESULTS AND DISCUSSION}

In order to study the kinetic behavior of the $\mathrm{Ni}-\mathrm{Ge}$ reaction in the $\mathrm{Ni} / \mathrm{Zr}$-interlayer/Ge system, isothermal annealing studies were conducted in a TEM equipped with a specimen-heating holder (EM-31050; JEOL Ltd.) at different temperatures. Isothermal in situ annealing experiments were carried out at $160^{\circ} \mathrm{C}, 180^{\circ} \mathrm{C}$, and $200^{\circ} \mathrm{C}$. Fig. 1 shows a cross-sectional HRTEM micrograph of a Ni film and a Zr-interlayer deposited on a Ge substrate observed at room temperature. The measured thicknesses of the $\mathrm{Ni}$ film and $\mathrm{Zr}$-interlayer were approximately $153 \AA$ and $10 \AA$, respectively.

The microstructural evolution of the interface between the $\mathrm{Zr}$-interlayer and the Ge substrate is displayed in a series of cross-sectional HR-TEM micrographs in Fig. 2 which was recorded using an UltraScan CCD camera during isothermal in situ annealing at $160^{\circ} \mathrm{C}$. The Ni germanide layer formed underneath the Zr-interlayer and grew to about $17 \AA$ in thickness when the temperature was raised from room temperature up to $160^{\circ} \mathrm{C}$ at the ramp rate of $16^{\circ} \mathrm{C} / \mathrm{min}$, and the TEM specimen was stabilized for recording the images. As 

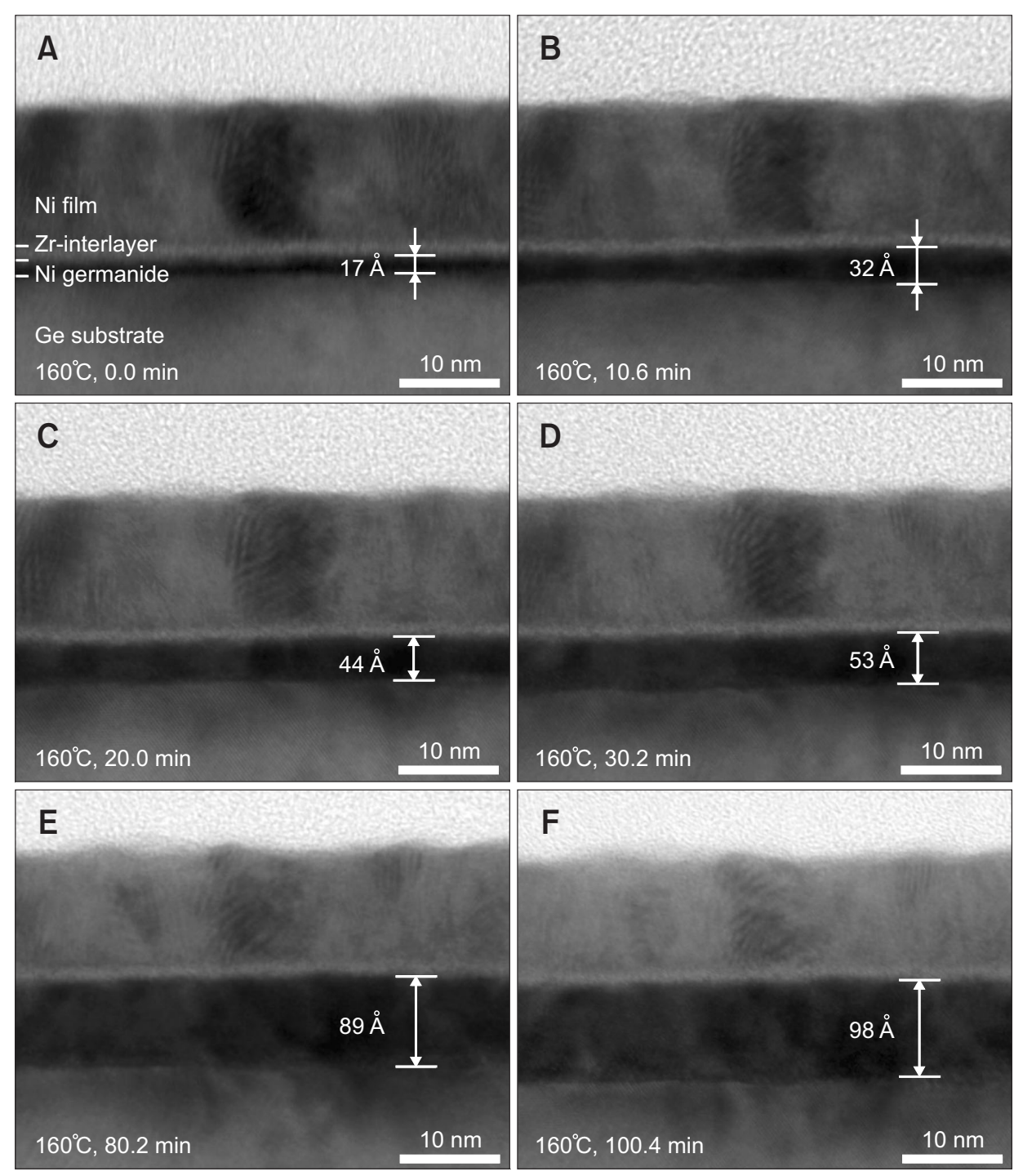

Fig. 2. (A-F) A series of cross-sectional transmission electron microscopy micrographs of $\mathrm{Ni} / \mathrm{Zr}$-interlayer/Ge system, which was recorded by UltraScan CCD camera (Gatan Inc.) during the isothermal in situ annealing at $160^{\circ} \mathrm{C}$ as a function of annealing time. the in situ annealing time increased, the Ni germanide layer grew thicker relatively uniformly, as shown in Fig. 2B-F. The thicknesses of the Ni germanide layer were measured to be 32 $\AA, 44 \AA, 53 \AA, 89 \AA$, and $98 \AA$ for annealing time of $10.6 \mathrm{~min}$, $20.2 \mathrm{~min}, 30.2 \mathrm{~min}, 80.2 \mathrm{~min}$, and $100.4 \mathrm{~min}$, respectively. The Ni germanide thickness as a function of the in situ annealing time was measured by taking the average of the thickness measured at 5 different locations. There was no noticeable change in the thickness of the $\mathrm{Zr}$-interlayer on top of the $\mathrm{Ni}$ germanide.

Similarly, isothermal in situ annealing experiments were also carried out at $180^{\circ} \mathrm{C}$ and $200^{\circ} \mathrm{C}$. Fig. 3 and 4 show the microstructural evolution of the interface between the $\mathrm{Zr}$ interlayer and the Ge substrate during isothermal in situ annealing at $180^{\circ} \mathrm{C}$ and $200^{\circ} \mathrm{C}$, respectively. Due to a limitation of ramp rate, the $\mathrm{Ni}$ germanide formed and grew until the temperature reached at the target temperature of isothermal in situ annealing experiment. Thus, the initial thickness of the Ni germanide at the starting point of the isothermal in situ annealing experiment increased as annealing temperature increased, for instance, $17 \AA$ for $160^{\circ} \mathrm{C}, 49 \AA$ for $180^{\circ} \mathrm{C}$, and $94 \AA$ for $200^{\circ} \mathrm{C}$. As shown in Fig. 3, for the isothermal in situ annealing experiment at $180^{\circ} \mathrm{C}$, the thicknesses of the $\mathrm{Ni}$ germanide layer were measured to be $49 \AA, 99 \AA, 131 \AA$, 154 $\AA, 176 \AA$, and $194 \AA$ for the annealing time of $0.0 \mathrm{~min}, 20.1$ min, $40.2 \mathrm{~min}, 60.2 \mathrm{~min}, 80.1 \mathrm{~min}$, and $99.8 \mathrm{~min}$, respectively. In the case of the isothermal in situ annealing experiment at $200^{\circ} \mathrm{C}$, as shown in Fig. 4, the thicknesses of the Ni germanide layer were measured to be $94 \AA, 118 \AA, 129 \AA, 166 \AA, 196 \AA$, and $220 \AA$ for the annealing time of $0.0 \mathrm{~min}, 5.0 \mathrm{~min}, 9.9 \mathrm{~min}$, $19.8 \mathrm{~min}, 29.8 \mathrm{~min}$, and $40.1 \mathrm{~min}$, respectively.

It was observed that an intermediate or intermixing layer was not formed in the interface between the $\mathrm{Zr}$-interlayer and the Ge substrate, and that the newly formed Ni germanide 


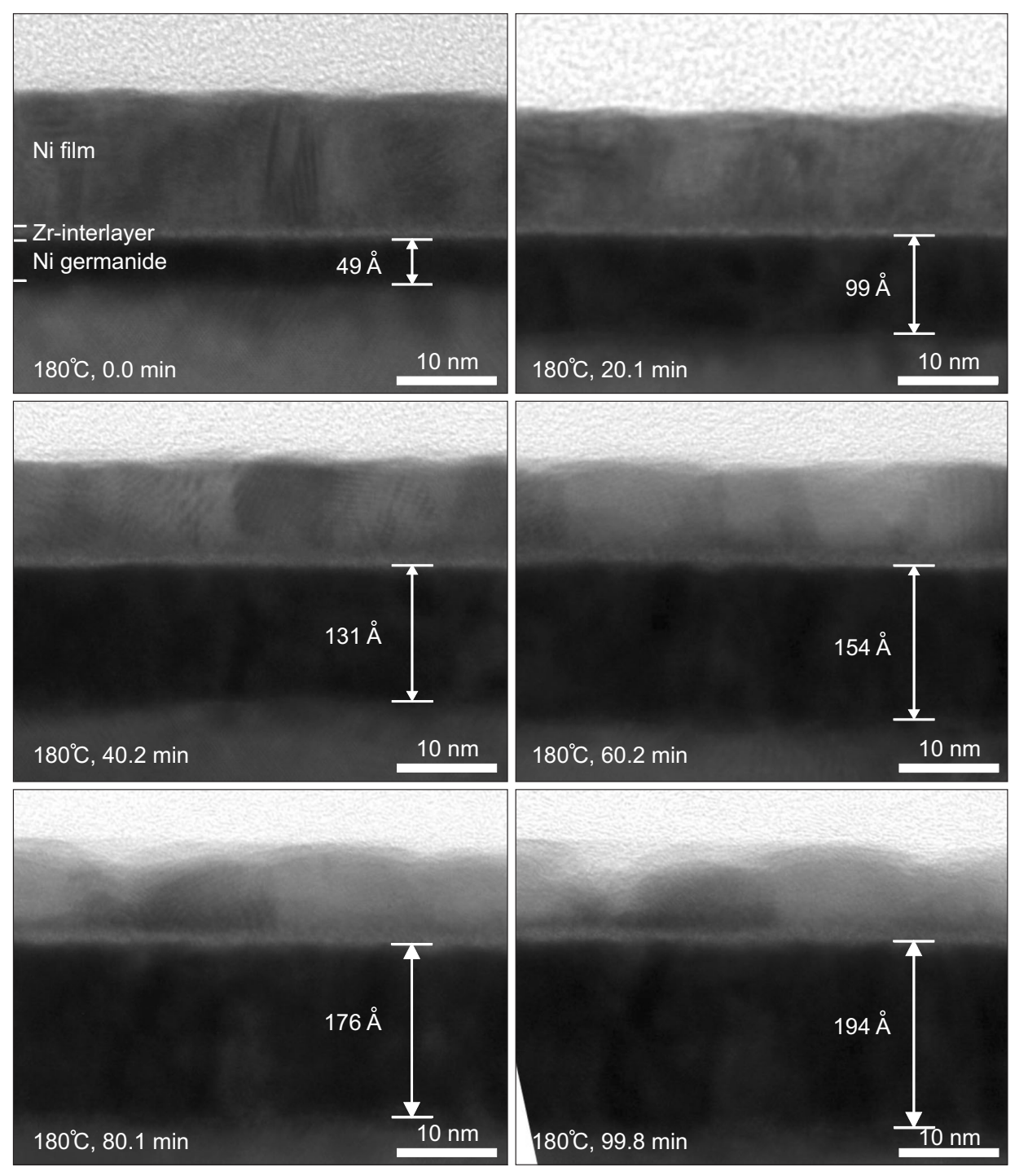

Fig. 3. A series of cross-sectional transmission electron microscopy micrographs of $\mathrm{Ni} / \mathrm{Zr}$-interlayer/Ge system, which was recorded by UltraScan CCD camera (Gatan Inc.) during the isothermal in situ annealing at $180^{\circ} \mathrm{C}$ as a function of annealing time. layers that grew during in situ annealing at these temperatures were more uniform compared with those in $\mathrm{Ni} / \mathrm{Ge}$ and $\mathrm{Ni} /$ Ta-interlayer/Ge systems in our previous study (Lee et al., 2015). In the case of the Ni/Ta-interlayer/Ge system where the Ta-interlayer is applied instead of the $\mathrm{Zr}$-interlayer in order to improve the thermal stability of the Ni germanide layer, in contrast, it was reported that the amorphous $\mathrm{Ni}$ Ta-Ge ternary layer was formed between the Ta-interlayer and the Ge substrate, and then, the ternary amorphous layer became thinner and the $\mathrm{Ni}$ germanide layer grew thicker with increasing annealing time.

The thickness of the Ni germanide layer as determined from the isothermal in situ annealing experiments is plotted in Fig. 5 as a function of the annealing time. It shows the kinetic data for the growth of $\mathrm{Ni}$ germanide in the $\mathrm{Ni} / \mathrm{Zr}$-interlayer/ Ge system with annealing time during isothermal in situ annealing at $160^{\circ} \mathrm{C}, 180^{\circ} \mathrm{C}$, and $200^{\circ} \mathrm{C}$. Since a diffusion- controlled reaction is typically observed in this system, the activation energy can be calculated from the isothermal in situ annealing data at three different temperatures.

For the diffusion-controlled reaction (Cotts et al., 1986; DeAvillez et al., 1990),

$$
X \frac{d x}{d t}=K
$$

where $X$ is the thickness of the Ni germanide layer and $K$ is the parabolic reaction rate constant. By integrating this equation from $X_{i}$, the initial thickness of the Ni germanide layer, to $X_{t}$, the thickness of the Ni germanide layer after annealing at a temperature $T$ for time $t$, we obtain the following equation:

$$
\frac{X^{2}{ }_{t} X^{2}}{2 t}=K
$$



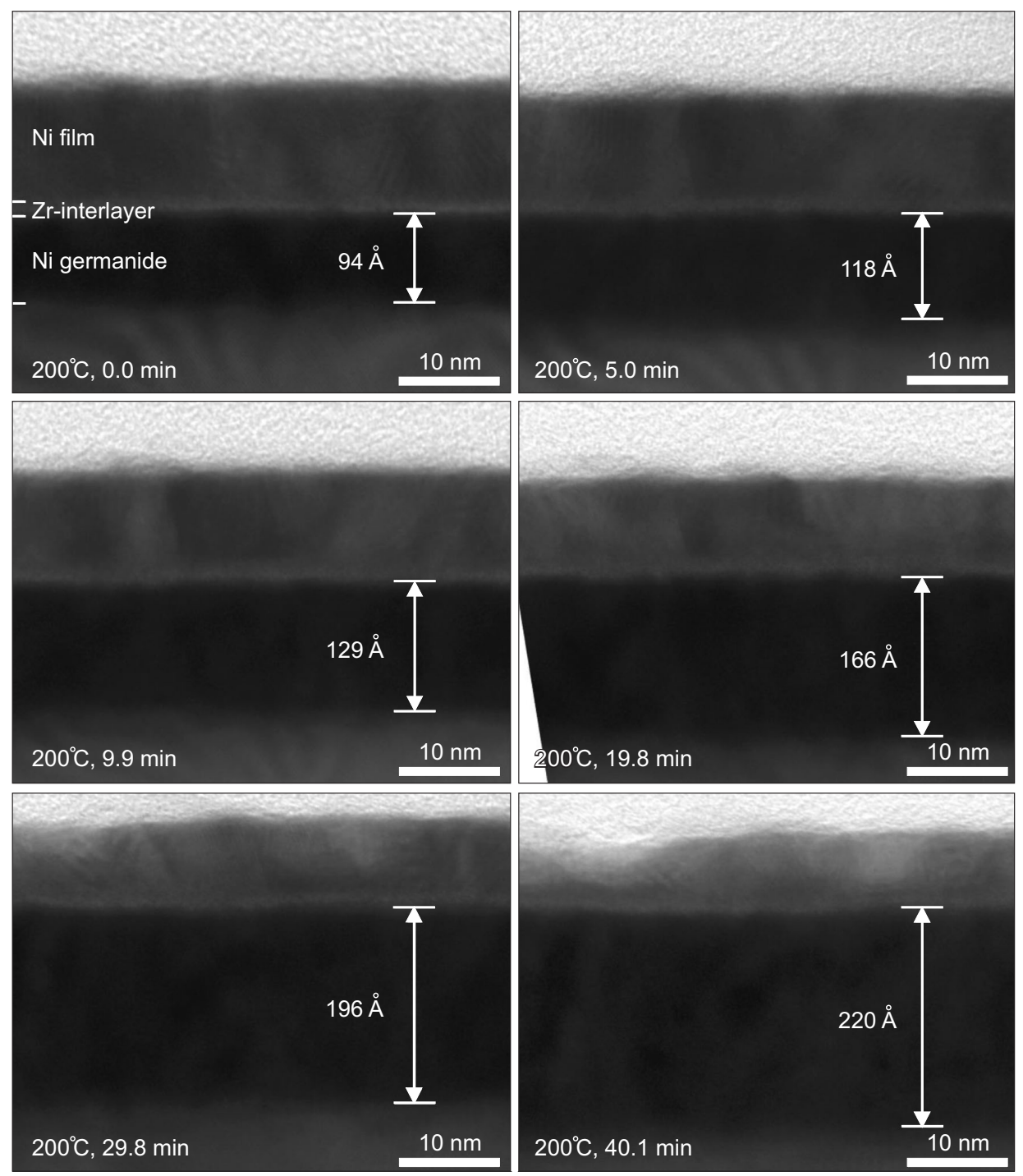

Fig. 4. A series of cross-sectional transmission electron microscopy micrographs of $\mathrm{Ni} / \mathrm{Zr}$-interlayer/Ge system, which was recorded by UltraScan CCD camera (Gatan Inc.) during the isothermal in situ annealing at $200^{\circ} \mathrm{C}$ as a function of annealing time.
Using the data obtained at three different temperatures, the values for $K$ were calculated. The growth of the Ni germanide in the $\mathrm{Ni} / \mathrm{Zr}$-interlayer/Ge system adheres to a parabolic rate law, as shown in Fig. 5. The activation energy barrier can be derived by using the following Arrhenius equation:

$$
K=A \exp \left[-\frac{E_{a}}{R T}\right]
$$

Fig. 6 shows an Arrhenius plot of the natural log of the parabolic reaction rate constant, $\left(X_{t}^{2}-X_{i}^{2} / 2 t\right)$ versus $1 /$ absolute temperature using the data in Fig. 5. From the slope of this plot the activation energy for the growth of the Ni germanide layer in the $\mathrm{Ni} / \mathrm{Zr}$-interlayer/Ge system was found to be $1.04 \pm 0.04 \mathrm{eV}$. The derived activation energy barrier for the growth of the $\mathrm{Ni}$ germanide layer in the $\mathrm{Ni} / \mathrm{Zr}$-interlayer/ Ge system was approximately $0.12 \mathrm{eV}$ higher than that in the
$\mathrm{Ni} / \mathrm{Ge}$ system. In the case of the $\mathrm{Ni} / \mathrm{Ta}$-interlayer/Ge system where the activation energy barrier is $0.21 \mathrm{eV}$ higher than that in the Ni/Ge system, the high activation energy barrier can be attributed to the formation of a ternary amorphous layer that observed in the interface between the Ta-interlayer and the Ge substrate in the initial stages of the isothermal in situ annealing experiment (Lee et al., 2015). Thus, the higher activation energy barrier for the formation and growth of $\mathrm{Ni}$ germanide in the Ni/Ta-interlayer/Ge system was caused by the limited diffusion of $\mathrm{Ni}$ atoms through not only the Tainterlayer, but also the ternary amorphous layer that remained between the Ta-interlayer and the newly formed Ni germanide layer.

For the Ni/Zr-interlayer/Ge system, on the other hand, no intermediate or intermixing layer between the $\mathrm{Zr}$-interlayer and Ge substrate was formed in the ex situ (not shown) and in situ annealing experiments, and thus the Ni germanide was 
A

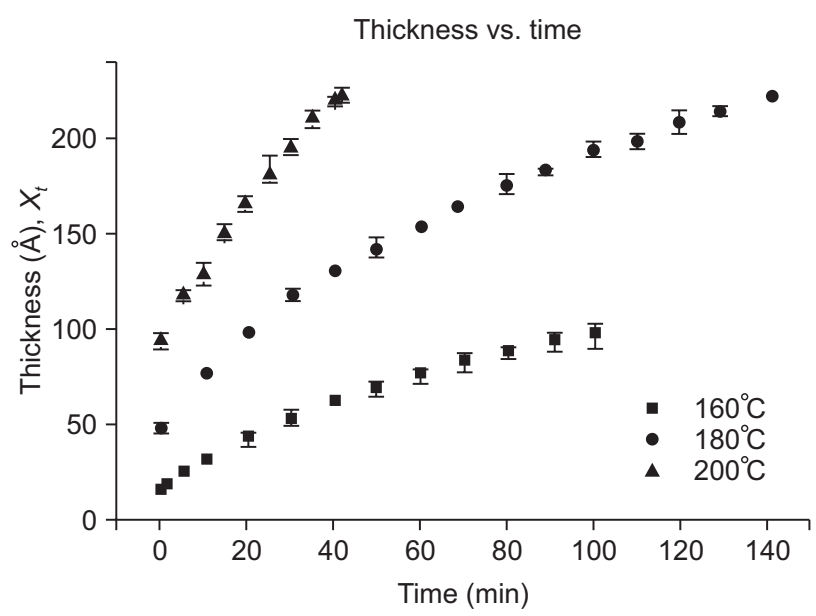

B

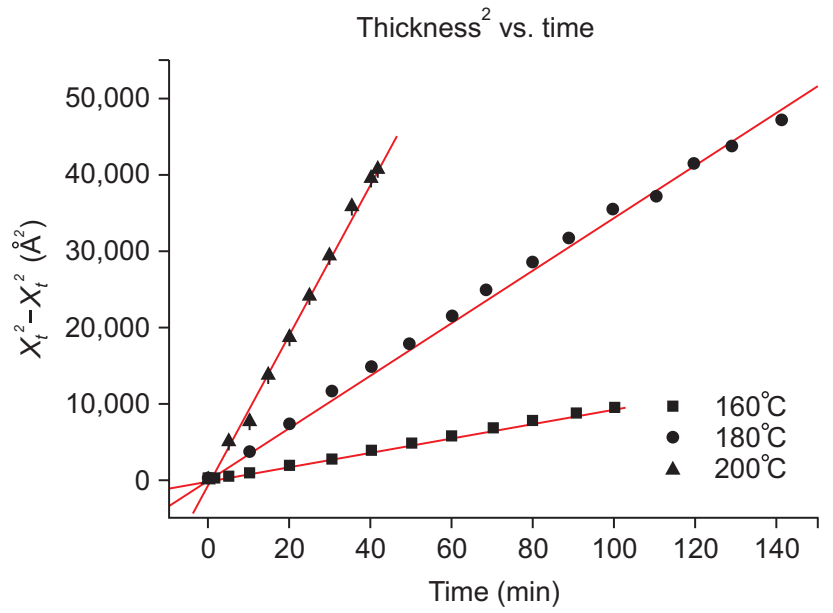

Fig. 5. Plots of the thickness of the Ni germanide layer in the Ni/Zr-interlayer/Ge system measured from the cross-sectional transmission electron microscopy micrographs as a function of the annealing time at three different temperatures of $160^{\circ} \mathrm{C}, 180^{\circ} \mathrm{C}$, and $200^{\circ} \mathrm{C}$. (A) Thickness versus time. (B) Thickness ${ }^{2}$ versus time.

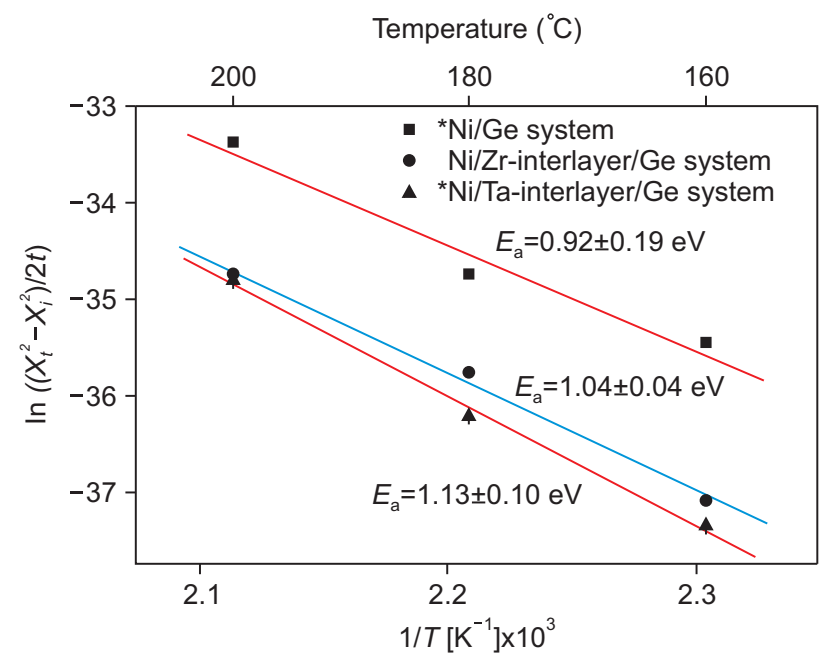

Fig. 6. Arrhenius plot of the growth rate of the Ni germanide layer in the $\mathrm{Ni} / \mathrm{Ge}, \mathrm{Ni} / \mathrm{Ta}$-interlayer/Ge and $\mathrm{Ni} / \mathrm{Zr}$-interlayer/Ge systems at three different temperatures of $160^{\circ} \mathrm{C}, 180^{\circ} \mathrm{C}$, and $200^{\circ} \mathrm{C}$. The activation energy for the $\mathrm{Ni} / \mathrm{Zr}$-interlayer/Ge system was calculated to be $1.04 \pm 0.04 \mathrm{eV}$. *The data for $\mathrm{Ni} / \mathrm{Ge}$ and $\mathrm{Ni} / \mathrm{Ta}$-interlayer/Ge systems obtained from Lee et al. (2015) (Sci. Adv. Mater. in print).

formed and grew uniformly due to Ni diffusion through the diffusion path created in the amorphous $\mathrm{Zr}$-interlayer during the annealing process in the absence of any intermetallic compounds. Therefore, the activation energy for the growth of the Ni germanide layer in the Ni/Zr-interlayer/Ge system was slightly higher than that in the $\mathrm{Ni} / \mathrm{Ge}$ system and a little lower than that in the Ni/Ta-interlayer/Ge system. However, in the previous in situ annealing experiments for the thick $\mathrm{Zr}$ interlayer ( $>3 \mathrm{~nm}$ ) (not shown), the germanidation reaction did not occur for temperatures up to $500^{\circ} \mathrm{C}$. It is believed that the formation of the Ni germanide was caused by the diffusion of $\mathrm{Ni}$ atoms through the $\mathrm{Zr}$-interlayer, which is affected by the thickness of the Zr-interlayer. The process of the formation and growth of the Ni germanide in the Ni/Zrinterlayer/Ge system discussed above is briefly displayed in the schematic diagram shown in Fig. 7.

\section{SUMMARY}

This study examined the reaction kinetics of the growth of $\mathrm{Ni}$ germanide in the $\mathrm{Ni} / \mathrm{Zr}$-interlayer/Ge systems using isothermal in situ annealing in a TEM and compared with the $\mathrm{Ni} / \mathrm{Ge}$ and Ni/Ta-interlayer/Ge systems. The reaction in the $\mathrm{Ni} / \mathrm{Zr}$-interlayer/Ge system was determined to be diffusion controlled with an activation energy of $1.04 \pm 0.04 \mathrm{eV}$, and depended on the square root of the time. This value for the activation energy barrier is slightly higher than that of the $\mathrm{Ni} /$ Ge system and a little lower than that of the Ni/Ta-interlayer/ Ge system. For the Ni/Ta-interlayer/Ge system, the increase in activation energy was due to the combination of the Tarich layer and the ternary amorphous layer formed in the initial stage of the annealing process. In contrast, the reaction kinetics in the Ni/Zr-interlayer/Ge system were affected only by the $\mathrm{Zr}$-interlayer. This study demonstrates that the kinetic data points can be correlated with the microstructural change at each time in the in situ annealing TEM experiment.

\section{CONFLICT OF INTEREST}

No potential conflict of interest relevant to this article was reported. 

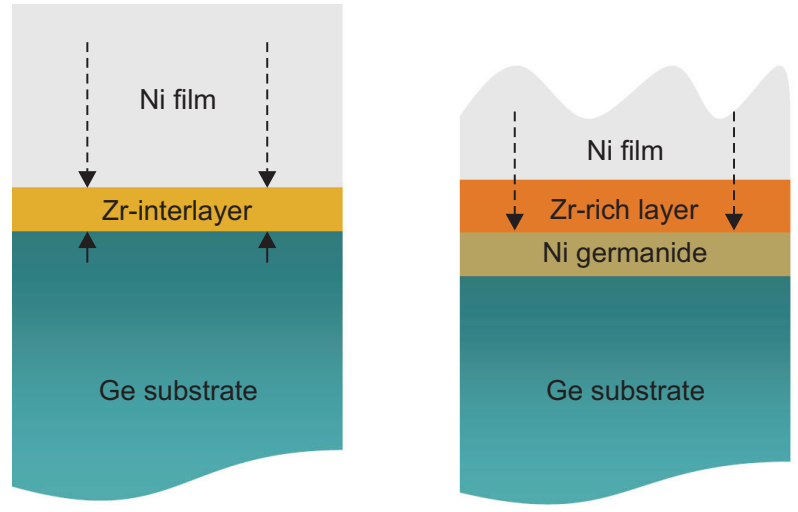

\section{ACKNOWLEDGMENTS}

The authors are grateful for the technical support provided

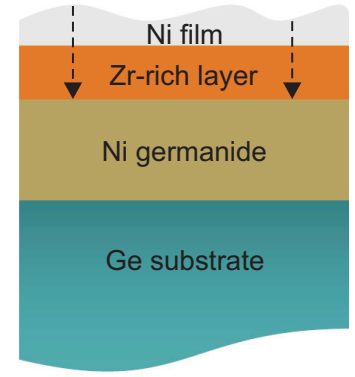

Fig. 7. Schematic diagrams of the process of the formation and growth of the $\mathrm{Ni}$ germanide in the $\mathrm{Ni} / \mathrm{Zr}$-interlayer/Ge systems.

by the Cooperative Center for Research Facilities (CCRF) at Sungkyunkwan University.

\section{REFERENCES}

Balakrisnan B, Tan C C, Liew S L, Lim P C, Goh G K, Foo Y L, and Chi D Z (2005) Texture of NiGe on Ge(001) and its evolution with formation temperature. Appl. Phys. Lett. 87, 241922.

Cotts E J, Meng W J, and Johnson W L (1986) Calorimetric study of amorphization in planar, binary, multilayer, thin-film diffusion couples of Ni and Zr. Phys. Rev. Lett. 57, 2295-2298.

DeAvillez R R, Clevenger L A, Thompson C V, and Tu K N (1990) Quantitative investigation of titanium/amorphous-silicon multilayer thin film reactions. J. Mater. Res. 5, 593-600.

Hsu S L, Chien C H, Yang M J, Huang R H, Leu C C, Shen S W, and Yang T H (2005) Study of thermal stability of nickel monogermanide on single- and polycrystalline germanium substrates. Appl. Phys. Lett. 86, 251906.

Lee J W, Bae J H, Kim T H, Kim H, Min S H, Shin K, Lee J H, Song J I, and Yang C W (2015) Kinetics of the Ni/Ta-interlayer/Ge reactions studied by in situ transmission electron microscopy. Sci. Adv. Mater. in print.

Lee J W, Bae J H, Park M H, Kang H B, Kim H, and Yang C W (2008) Microstructural evolution of nickel-germanide in the $\mathrm{Ni}(1-\mathrm{x}) \mathrm{Tax} / \mathrm{Ge}$ systems during in situ annealing. J. Vac. Sci. Technol. A 26, 688-691.

Lee J W, Kim H K, Bae J H, Park M H, Kim H, Ryu J, and Yang C W (2013) Enhanced morphological and thermal stabilities of nickel germanide with an ultrathin tantalum layer studied by ex situ and in situ transmission electron microscopy. Microsc. Microanal. 19, 114-118.

Lee J W, Kwak K H, Kim H, Yang C W, Lee D, and Ko D H (2007) In-situ annealing study on the thermal stability of nickel germanides. J. Korean Phys. Soc. 50, 677-680.

Zhang Q, WU N, Osipwicz T, Bear L K, and Zhu C (2005) Formation and thermal stability of nickel germanide on germanium substrate. Jpn. J. Appl. Phys. 44, L1389-L1391.

Zhang Y Y, Oh J, Bae T S, Zhong Z, Li S G, Jung Y S, Park K Y, Lee G W, Wang J S, Majhi P, Lee B H, Tseng H H, Jeong Y H, and Lee H D (2008) Phase separation of Ni germanide formed on a Ge-on-Si structure for Ge MOSFETs. Electrochem. Solid-State Lett. 11, H1-H3.

Zhu S and Nakajima A (2005) Annealing temperature dependence on nickel-germanium solid-state reaction. Jpn. J. Appl. Phys. 44, L753-L755. 